\section{Mitocellular communication: Shaping health and disease}

\author{
Adrienne Mottis*, Sébastien Herzig*, Johan Auwerx†
}

Throughout the animal kingdom, mitochondria are the only organelles that retain their own genome and the transcription and translation machineries that are all essential for energy harvesting. Mitochondria have developed a complex communication network, allowing them to stay in tune with cellular needs and nuclear transcriptional programs and to alleviate mitochondrial dysfunction. Here, we review recent findings on the wide array of mechanisms that contribute to these mitocellular communication networks, spanning from well-studied messenger molecules to mitonuclear genetic interactions. Based on these observations and developments, we advocate a broad and inclusive view on mitocellular interactions, which can have profound impacts on physiological, pathological, and evolutionary processes.

M itochondria are essential components of eukaryotic cells. They are not, however, isolated organelles; mitochondria exchange molecules-from ions and small metabolites to proteins and lipids-with the rest of the cell and the organism. Mitochondria originate from the endosymbiotic interaction between an $\alpha$-proteobacterium and its host cell $>1$ billion years ago. During evolution, most genes encoded in the mitochondrial genome were transferred to the nucleus, leaving only a few protein-coding genes in the vestigial circular mitochondrial DNA (mtDNA); therefore, mitochondria and nucleus need to communicate to ensure optimal cellular function. Moreover, mitochondria actively influence other cellular components such as the lysosomes, the endoplasmic reticulum (ER), and cytosolic pathways, creating a mitocellular communication network that is based on a variety of signals and cues, akin to different languages (Fig. 1A)

Mitochondria can use such signals to adapt to various stresses, leading to a beneficial outcome in a phenomenon termed mitohormesis. In other cases, deleterious consequences for the cell or the organism ensue. Understanding the differences between adaptive and maladaptive responses to stress is key to leveraging mitochondrial communication to maintain health at the cellular or organismal level. The cellular response to mitochondrial dysfunction ranges from up-regulation of chaperones and proteases to improve proteostasis to the degradation of mitochondria by mitophagy. During aging, mitochondria lose function and mitocellular communication pathways break down; strategies to maintain and invigorate mitochondrial function therefore increase the health span and life span.

Laboratory of Integrative Systems Physiology, Ecole Polytechnique Fédérale de Lausanne (EPFL), CH-1015 Lausanne, Switzerland.

*These authors contributed equally to this work.

†Corresponding author. Email: admin.auwerx@epfl.ch
Small molecules as mitochondrial messengers As the focal point of cellular metabolism, it comes as no surprise that metabolites play a prime role in signaling changes in mitochondrial activity to other cellular compartments (1) (Fig. 1B).

\section{AMP}

Because mitochondria are the major site of adenosine $5^{\prime}$-triphosphate (ATP) generation, ATP levels are a sensitive signal of mitochondrial health. In the wake of decreases in ATP production, ongoing energy utilization leads to an increase in the cellular adenosine $5^{\prime}$ monophosphate (AMP)/ATP ratio, which activates a specialized signaling pathway, the AMP-activated protein kinase (AMPK) pathway. Active AMPK phosphorylates key enzymes and regulatory nodes involved in various metabolic pathways, such as lipid and glucose metabolism, mitochondrial dynamics, autophagy, and protein synthesis, to restore energy balance (2). In addition, AMPK also leads to transcriptional adaptations to energy stress, allowing cells to further match their metabolism to mitochondrial signals, such as decreased ATP levels. Exploiting these mitohormetic energetic stresssignaling pathways through activation of AMPK holds promise for the management of several diseases.

\section{$N A D^{+}$}

The nicotinamide adenine dinucleotide $\left(\mathrm{NAD}^{+}\right) /$ $\mathrm{NADH}$ ratio is another indicator of the metabolic status of the mitochondria. $\mathrm{NAD}^{+}$is a cofactor for numerous metabolic reactions, but it also serves as a cosubstrate for enzymes such as CD38, poly-ADP-ribose polymerases, and the sirtuins, a family of seven protein deacetylases and deacylases localized in the nucleus (SIRT1, SIRT6, and SIRT7), cytosol (SIRT2), and mitochondria (SIRT3 to SIRT5). Changes in the abundance of $\mathrm{NAD}^{+}$directly affect the activity of the sirtuins in multiple cellular compartments, allowing the fine tuning of metabolism through the deacetylation of multiple metabolic regulators often linked to mitochondrial homeostasis (3). $\mathrm{NAD}^{+}$levels not only vary with metabolic activity, e.g., caloric restriction and high-fat diets, but also decrease during aging (4) and increase during exercise and upon pharmacological or nutraceutical interventions that increase $\mathrm{NAD}^{+}$levels. Elevated $\mathrm{NAD}^{+}$levels are linked to improved health span and life span in multiple model organisms (5). Therefore, $\mathrm{NAD}^{+}$levels and their commanding function on the activity of the sirtuins are key contributors to sensing and communicating the mitochondrial metabolic status to other cellular compartments.

\section{Oxygen}

Mitochondria require oxygen for ATP generation, so low oxygen levels, or hypoxia, influence mitochondrial function. In return, mitochondria consume oxygen and thus potentially reduce local oxygen concentration. Cells are capable of sensing low oxygen through prolyl hydroxylase domain proteins (PHDs) that stabilize hypoxia-inducible factor (HIF)- $1 / 2 \alpha$. HIF- $1 \alpha$ stabilization mediates some of the deleterious effects of decreased $\mathrm{NAD}^{+}$levels and sirtuin activity on mitochondrial function in aged mice, a process that is reversed by boosting $\mathrm{NAD}^{+}$levels or by caloric restriction (6). Stabilization of HIF-1 $\alpha$ in vivo by knocking out the VHL (Von Hippel-Lindau) gene in zebrafish or by chronic hypoxia in mice improves the viability of mitochondrial disease models (7). This sounds counterintuitive at first, as restricting oxygen availability may further impair mitochondrial function. However, the fact that hypoxia and HIF signaling allow cells to adapt to lower mitochondrial function and thus decrease the strain imposed on dysfunctional mitochondria explains this apparent conundrum.

\section{ROS}

Mitochondrial dysfunction can also generate toxic by-products such as reactive oxygen species (ROS). Under physiological conditions, ROS act as signaling molecules to control hypoxic responses, immunity, and stem cell function (8). Several lines of evidence also point to ROS as a mediator of longevity downstream of mitochondrial modulation. ROS produced by mitochondria stabilizes HIF-1 $\alpha$ (9), which is important for life-span extension induced by mitochondrial inhibition in Caenorhabditis elegans and replicative life span of mammalian cells (10). Thus, mitochondrial ROS and modulation of HIF signaling participate in the mitohormetic response.

\section{Metabolites}

Metabolites that are produced and used inside mitochondria, most notoriously tricarboxylic acid (TCA) cycle components, also signal mitochondrial health. For example, acetyl-coenzyme 
A

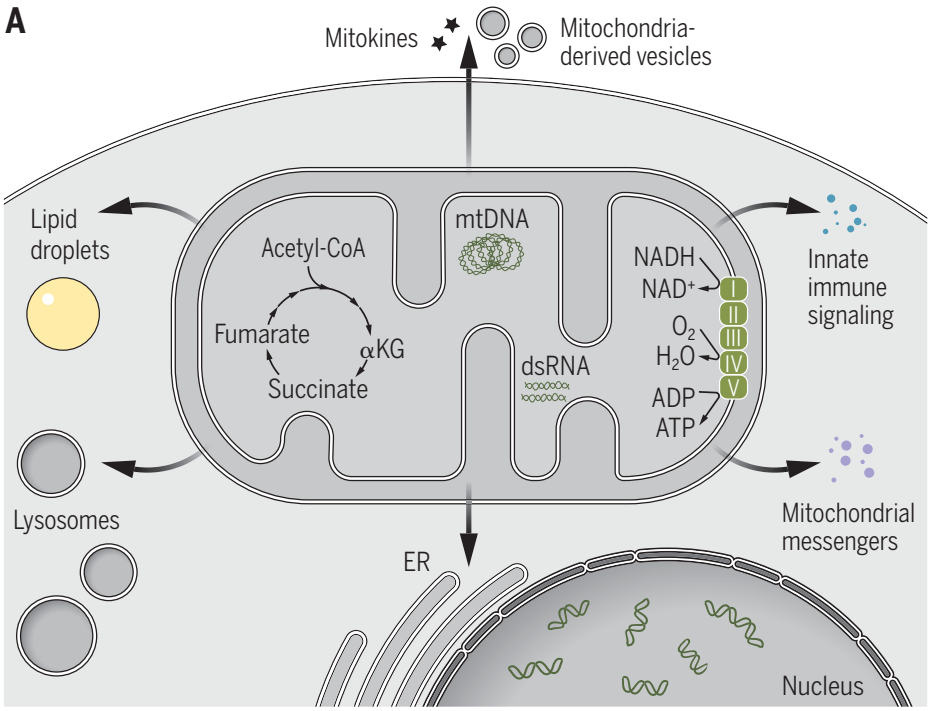

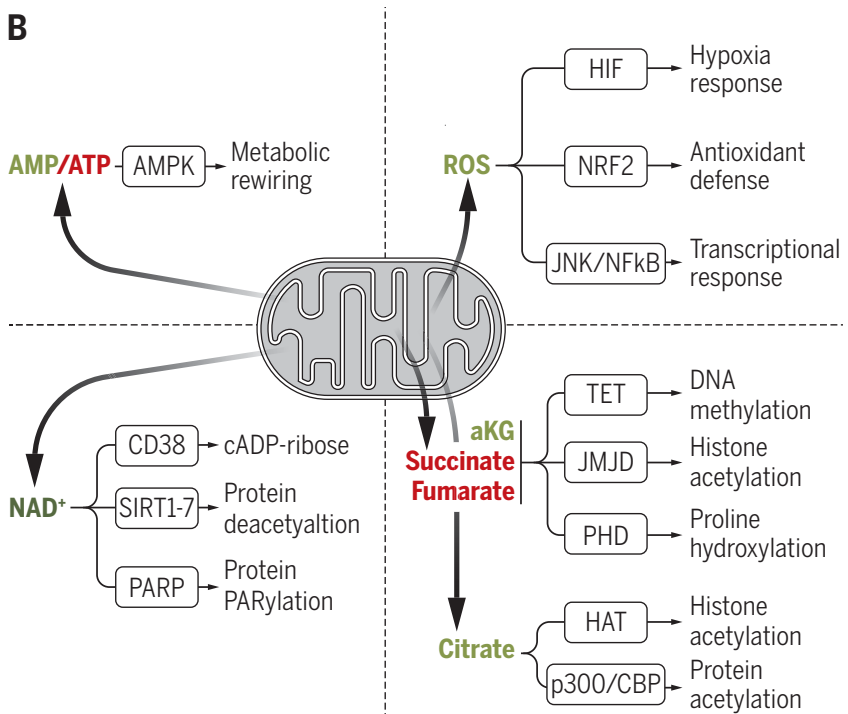

Fig. 1. Overview of mitocellular interactions. (A) Mitochondria interact with the cellular environment through physical contacts, signaling molecules, and extracellular signals. (B) Mitochondrial metabolic languages. Various mitochondrial metabolites are sent by the mitochondria and translated by cellular signaling pathways into an appropriate response. Green indicates metabolites that activate the pathway and red indicates inhibitory signals.

A (acetyl-CoA), which is produced from various sources inside mitochondria-e.g., pyruvate, amino acids, and fatty acids-enters the TCA cycle to generate citrate. Citrate can either continue within the TCA cycle or exit mitochondria and produce acetyl-CoA, which is an essential cosubstrate for the acetyl transferases to fuel histone and protein acetylation (11). Variations in acetyl-CoA are thus mitochondrial signals that can modulate broad gene expression programs. $\alpha$-Ketoglutarate $(\alpha \mathrm{KG})$, succinate, and fumarate also act as signaling molecules. $\alpha \mathrm{KG}$ serves as a cofactor for dioxygenase enzymes such as the Jumonji C domain-containing histone demethylases (JMJDs), TET (Ten-Eleven Translocation) DNA demethylases, and PHDs; conversely, succinate and fumarate inhibit these $\alpha \mathrm{KG}$-dependent enzymes (12). Dioxygenases can also be inhibited by 2-hydroxyglutarate (2-HG), a metabolite similar in structure to $\alpha \mathrm{KG}$. Whereas D2-HG is produced by isocitrate dehydrogenase (IDH)-1/2 mutant cancer cells, mitochondria produce L2-HG during hypoxia (13) or respiratory chain inhibition (14) and regulate metabolism through epigenetic modifications.

\section{Proteotoxic stress: Transcriptional and epigenetic regulation}

The mitochondrial stress response (MSR) orchestrates the remodeling of gene expression programs after mitochondrial proteotoxic stress. The mitochondrial unfolded protein response $\left(\mathrm{UPR}^{\mathrm{mt}}\right)$, an arm of the MSR that signals proteotoxic stress to induce an adaptive response aimed at resolving protein-folding stress, has been particularly well studied in C. elegans (15), although recent work suggests its conservation in vertebrates $(16-18)$. The
MSR is induced by toxins or mitochondrial dysfunctions typified by a stoichiometric mismatch between nuclear DNA (nDNA)- and mtDNA-encoded oxidative phosphorylation complex (OXPHOS) subunits, resulting in the accumulation of orphan, unassembled OXPHOS subunits in the mitochondria. Most $\mathrm{UPR}^{\mathrm{mt}}$ triggering insults are accompanied by a drop in mitochondrial protein import capacity. The localization of ATFS-1 (activating transcription factor associated with stress-1), the master transcriptional regulator of the C. elegans $\mathrm{UPR}^{\mathrm{mt}}$, is also controlled by mitochondrial protein import. Whereas ATFS-1 is normally imported in healthy mitochondria, mitochondrial stress blocks its import, favoring its nuclear localization (19). ATFS-1 induces UPR ${ }^{\mathrm{mt}}$ genes, including chaperones, proteases, detoxification enzymes, and mediators of metabolic reprograming. Worm UPR ${ }^{\mathrm{mt}}$ involves active epigenetic reprogramming; chromatin decompaction and induction of $\mathrm{UPR}^{\mathrm{mt}}$ genes is mediated by the H3K27 demethylases JMJD-3.1 and JMJD-1.2, and a global chromatin compaction is achieved by the histone lysine transferase MET-2, assisted by LIN-65, to avoid transcription of nonessential genes in stressful conditions (20,21) (Fig. 2A). Epigenetic remodeling caused by mitochondrial stress extends the life span (20) and is transmitted over four generations through histone $\mathrm{H} 3 \mathrm{~K} 4$ methylation (22). JMJD expression also correlates with life span and $\mathrm{UPR}^{\mathrm{mt}}$ genes in the BXD mouse genetic reference population, indicating that this link between epigenetic remodeling and life extension may be conserved in mammals (20).

In mammalian cells, several mitochondrial insults, including proteotoxic stress and ROS, signal through the integrated stress response (ISR) $(23,24)$. The activated GCN2 kinase is one of the kinases known to phosphorylate the translation initiation factor eIF2 $\alpha$ (25). eIF2 $\alpha$ phosphorylation generally leads to a global slowdown of cytosolic, cap-dependent translation, transitioning to stress-induced alternative translation, a mechanism used by various stressors (24). This favors the translation of stress transcripts containing upstream open reading frames, including the Atf4, Atf5, and Chop transcription factors that coordinate a gene expression program considered the mammalian equivalent of $\mathrm{UPR}^{\mathrm{mt}}$ (Fig. 2A). It also suggests that signaling of the mammalian $\mathrm{UPR}^{\mathrm{mt}}$ is directly connected to and partially dependent on the same actors that govern cytosolic proteostasis, a functional link supported by the mitochondria-to-cytosol stress response in C. elegans (24). Another mediator of the mammalian MSR was recently shown to adopt a mechanism recalling ATFS-1 signaling in C. elegans. After mitochondrial membrane depolarization, G-Protein Pathway Suppressor 2 (GPS2) translocates from mitochondria to the nucleus, where it binds to target promoters and induces H3K9 demethylation, enabling transcription of mitochondrial- and stress-responsive genes, which partially overlap with ATF4 targets (26).

Mitochondrial stress signaling in both invertebrates and vertebrates, albeit different, shares similarities: in both cases, it is dependent on the ATF transcription factor family and involves the modulation of H3 methylation and the mitonuclear translocation of transcription factors. Together with the antioxidant response, the MSR is one of the major mitochondrial pathways mediating mitohormesis. 
A

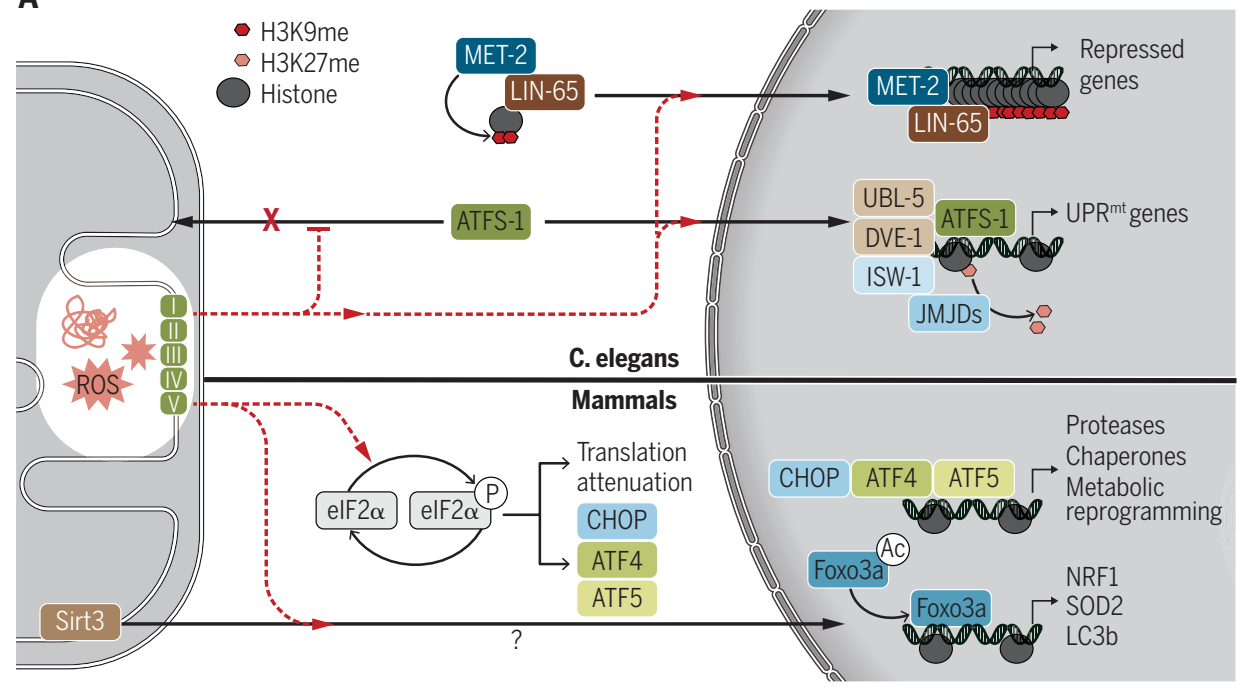

B

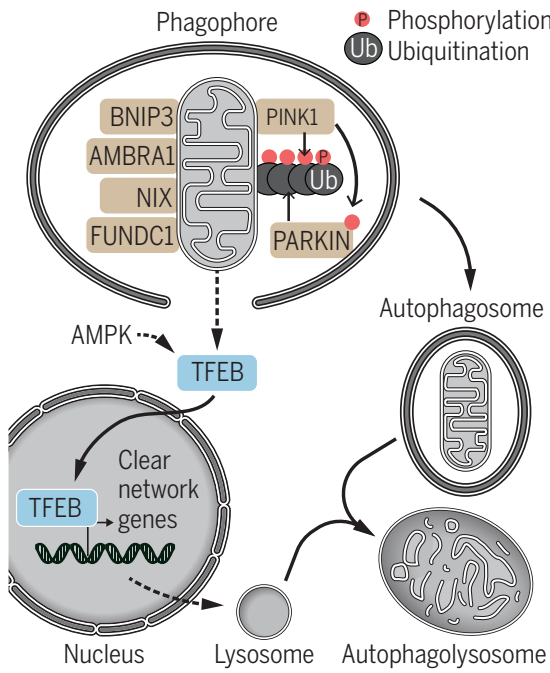

\section{C (i) mitoTAD (constant} monitoring of mitochondrial import)

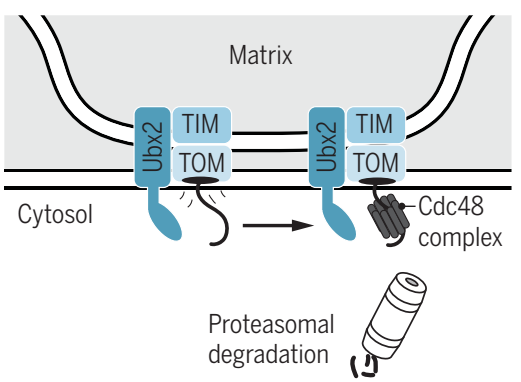

(ii) Stress-induced inhibition of protein import

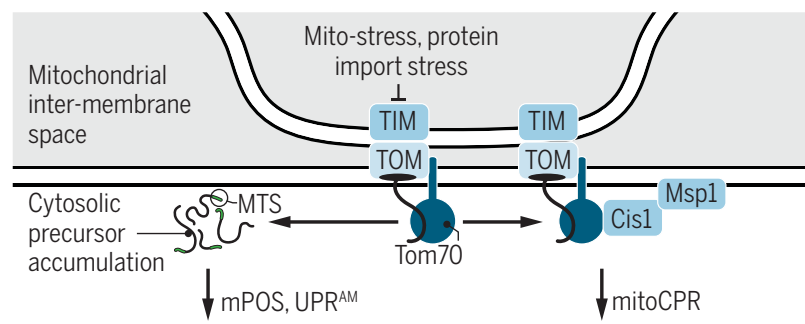

- Cytosolic proteostasis genes -Inhibition of translation -Proteasomal degradation - (iii) Mitochondria As Guardian In the Cytosol (MAGIC)

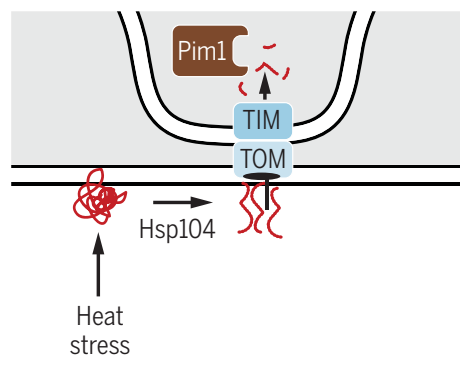

Fig. 2. Main mechanisms of translational control of the MSR in C. elegans and mammals. (A) A variety of mitochondrial stressors (unfolded proteins, electron transport chain loss-of-function, ROS) can induce nuclear translocation of transcription factors and changes in chromatin conformation and gene expression. The top part of the panel summarizes MSR signaling in C. elegans, and the bottom part focuses on mammalian cells. (B) Overview of the mitophagy pathway mediating the removal of mitochondria. Cross-talk between mitochondria and lysosomes through regulation of lysosomal genes is essential for efficient execution of MSR pathways such as mitophagy. (C) Quality control pathways involving the mitochondrial protein import machinery. (i) In yeast, mitochondrial protein translocation-associated degradation (mitoTAD) is mediated by Ubx2, which recruits the AAA-ATPase
Cdc48 to prevent clogging of the TOM complex with mitochondrial precursor proteins. (ii) Mitochondrial stress slows down mitochondrial protein import and leads to the accumulation of precursors in the cytosol. This "mitochondrial precursor overaccumulation stress" (MPOS) and the UPR activated by mistargeting of proteins (UPR ${ }^{\mathrm{AM}}$ ) triggers programs to restore cytosolic proteostasis, inhibit translation, and activate the proteosome. Protein import stress activates the mitochondrial-compromised protein import response (mitoCPR). It induces gene expression and recruits the AAA-ATPase Msp1 through Tom70 and Cis1 to degrade precursors stalled in the TOM complex. (iii) Protein aggregates that form at mitochondrial surface upon heat shock are disaggregated by the chaperone Hsp104, imported by the TOM complex, and degraded by the LON protease Pim1.
As mentioned before, such adaptations are not always beneficial; the persistent induction of mitohormetic signature genes (e.g., antioxidant and mitophagy genes) by the SIRT3 arm of the UPR ${ }^{\mathrm{mt}}$ contributes to breast cancer invasiveness (17). The remodeling of mitochondrial signaling therefore appears to be a key step in malignancy, because mitohormesis allows tumor cells to resist oxidative stress and favors their survival.

\section{Lysosomes, ER, and cytosol}

Mitocellular communication also encompasses how mitochondria affect other organelles' function through direct contact and indirect signaling. Mitochondria physically interact with many other organelles to ensure proper cellular function (27). For example, mitochondria-associated ER membranes (MAMS) are sites of physical proximity of the ER and the mitochondrial outer membranes, allowing lipid and calcium exchanges and dictating how mtDNA is replicated and segregated, as well as when and where mitochondria divide (28).

Mitochondria also physically interact with lysosomes (29). They affect the regulation of lysosomal function, which is essential for au- tophagy and mitophagy, a process by which damaged mitochondria are removed (Fig. 2B) (30). Lysosomal function is regulated in part by the TFEB (transcription factor EB) family of transcription factors through induction of the CLEAR (Coordinated Lysosomal Expression and Regulation) network, a gene set involved in lysosome biogenesis and function. TFEB activity is modulated downstream of mitochondrial dysfunction by $\operatorname{AMPK}(31,32)$ and through an AMPK-independent, PINK1- and Parkindependent pathway that also requires the autophagy gene ATG5 (33). Thus, mitochondrial dysfunction signals to increase lysosomal 
function, which in turn ensures proper removal of damaged mitochondria. Lysosomal storage disorders are accompanied by mitochondrial dysfunction and, conversely, mitochondrial diseases are associated with impairments in lysosomal function (34).

This reciprocal regulation of mitochondrial quality by lysosomes and lysosomal function by mitochondrial quality plays a key role in neurodegeneration. Mutations in PINK1 and Parkin and impaired mitophagy are associated with familial forms of Parkinson's disease (PD). Mitochondrial deficiency also occurs in patients with $\beta$-amyloid diseases such as Alzheimer's disease (AD). An MSR gene signature typifies patients with $\mathrm{AD}$ and cognitive impairment, and activating two components of the MSR, $\mathrm{UPR}^{\mathrm{mt}}$ and mitophagy, in $\mathrm{AD}$ models delays protein aggregation and disease progression (35). From a therapeutic perspective, it is interesting that boosting mitophagy by administration of urolithin A improves mitochondrial and muscle function in C. elegans, mouse, rat (36), and humans (37); has beneficial effects in animal models of $\mathrm{AD}(38)$; and extends life span in C. elegans (36). Consistent with these observations, activation of lysosomal function reestablishes youthful proteostasis in old C. elegans oocytes immediately before fertilization, a phenomenon accompanied by improvement of mitochondrial parameters (39).

Proteostasis in the mitochondria is also intimately connected to cytosolic proteostasis (24). Despite uncoordinated transcription of nDNA- and mtDNA-encoded OXPHOS mRNAs, OXPHOS stoichiometry is maintained through coordinated protein synthesis (40). During mitochondrial stress in mammals, activation of cap-independent cytosolic translation and activation of the ISR is part of the defense system $(23,24)$. In yeast, mitochondrial protein translocation-associated degradation (mitoTAD) constitutively monitors mitochondrial protein import to prevent obstruction of the TOM (translocase of the outer membrane) import channel with mitochondrial protein precursors (41) [Fig. 2C(i)]. During mitochondrial stress, the impairment of protein import causes precursor proteins to accumulate in the cytosol, referred to as "mitochondrial precursor overaccumulation stress" (mPOS), leading to the induction of cytosolic genes promoting alternative cytosolic translation mechanisms and protein folding and degradation (24) [Fig. $2 \mathrm{C}(\mathrm{ii})]$. Cytosolic proteostasis is also restored by the UPR activated by mistargeting of proteins $\left(\mathrm{UPR}^{\mathrm{AM}}\right)$, which inhibits protein synthesis and activates the proteasome (24), and by the mitochondrial-compromised protein import response (mitoCPR), which removes stalled precursors accumulated on the mitochondrial surface (42) [Fig. 2C(ii)]. Conversely, mitochondria buffer cytosolic protein aggregate load by importing and degrading aggregation- prone proteins in normal physiological and heat-stress conditions in yeast (43) [Fig. 2C(iii)].

Mitochondria maintain a degree of specialization across tissues and even within the same cell, leading to the coexistence of several mitochondrial subpopulations $(44,45)$. In brown adipose tissue, peridroplet mitochondria are specialized and dedicated to lipid synthesis and droplet expansion; mediators of mitochondrial dynamics are pivotal in segregating these mitochondria pools (45). In mammalian breast tissue, stemness of daughter cells is conserved by asymmetric sorting of young mitochondria; Parkin and mitochondrial fission are necessary to confine old mitochondria to the perinuclear region of mother cells (46). Likewise, mitochondrial dynamics (fusion) determines the asymmetric distribution of mitochondria and the fate of mammary stem cells in the epithelial-mesenchymal transition (47).

\section{Nucleic acids: Linking mitochondria and inflammation}

Cells depend on pattern-recognition receptors and innate immune pathways to detect and contend with viral and bacterial components in the cytosol. Accommodating an endosymbiotic guest such as the mitochondria implies

\section{“...reciprocal regulation of mitochondrial quality by lysosomes and lysosomal function by mitochondrial quality plays a key role in neurodegeneration."}

a possible reactivity toward the components inherited from its bacterial ancestry. Accordingly, immunostimulation can ensue from the release of $\mathrm{N}$-formylated peptides or mtDNA, as well as of several nucleic acid species generated by mitochondria, such as double-stranded RNA (dsRNA) or DNA-RNA hybrids (48). A wide range of mitochondrial insults can lead to the presence of mtDNA in the cytosol or even in the circulation, which upon detection by Tolllike receptors (TLRs), NOD-like receptors (NLRs), or interferon (IFN)-stimulatory DNA receptors, triggers proinflammatory and type I IFN responses (48) involving the cGAS-STING pathway. This pathway is responsible for the inflammation observed in $\mathrm{PinkI}^{-/-}$and $\mathrm{Prkn}^{-/-}$ mice upon an exercise challenge and for the loss of dopaminergic neurons and motor defects in aged $\mathrm{Prkn}^{-1-} ;$ mutator mice, which accumulate mtDNA mutations (49). Therefore, mitophagy is key in preventing mitochondrial leakage and the resulting immunostimulation, which seems crucial in the pathogenesis of PD.
Cells also conserved immunity against highly unstable mitochondrial dsRNA, highlighting the pivotal role of the polynucleotide phosphorylase (PNPase), which limits dsRNA accumulation in the mitochondrial matrix and intermembrane space (50). Moreover, mtDNA synthesis is instrumental in activating the NLRP3 inflammasome: various NLRP3 activators induce the release of mtDNA, which amplifies initial NLRP3 priming (51). Together, these findings reinforce the contribution of mitochondria and mitochondrial quality control in immune regulation and warrant the study of mitochondrial contributions to pervasive inflammatory processes that are common in many age-related diseases. Mitochondria can, however, regulate innate and adaptive immunity through multiple mechanisms (52). As an example, disrupted mitophagy and the resulting presentation of mitochondrial antigens are responsible for autoimmunity and PDlike symptoms upon bacterial infection in the intestine of $\mathrm{Pink1}^{-/-}$mice (53).

\section{Mitokines: Mitochondrial hormones}

Mitochondria not only signal within the cell, but also communicate with distant tissues in a non-cell-autonomous manner through circulating molecules. Mitokines are nuclearencoded signaling molecules secreted by cells experiencing mitochondrial stress. They are thought to mediate metabolic adaptation of distant tissues by enhancing oxidative metabolism, lipolysis, and ketogenesis as a logical reaction to the energy crisis produced by mitochondrial dysfunction. In mammals, circulating levels of FGF21 (25) and GDF15 (54) are increased in mouse models with mitochondrial dysfunction in an ATF4- and CHOP-dependent manner, respectively, as well as in patients with mitochondrial diseases (15). In C. elegans, the Wnt/EGL-20 ligand is the best characterized neuronal mitokine and is dependent on serotonin secretion to transduce an MSR to distant tissues (55). Mitochondrial-derived peptides, which are encoded within alternative reading frames in the mitochondrial genome, can also act as signaling factors to achieve systemic cytoprotective effects and stress resistance by improving insulin sensitivity and adiposity (56).

Finally, one can speculate about mitochondria communicating with distant tissues through mitochondria-derived vesicles containing mtDNA or mitochondrial components $(57,58)$ or even through intercellular transfer of mitochondria (59). These processes can be triggered upon acute neuronal or cardiac stress $(60,61)$, leading to positive feedback and cytoprotection of the recipient cells. Worm and mouse neuronal cells can also transfer mitochondria to outsource their degradation to other cells $(62,63)$. Although the signaling role of these mechanisms has not yet been 


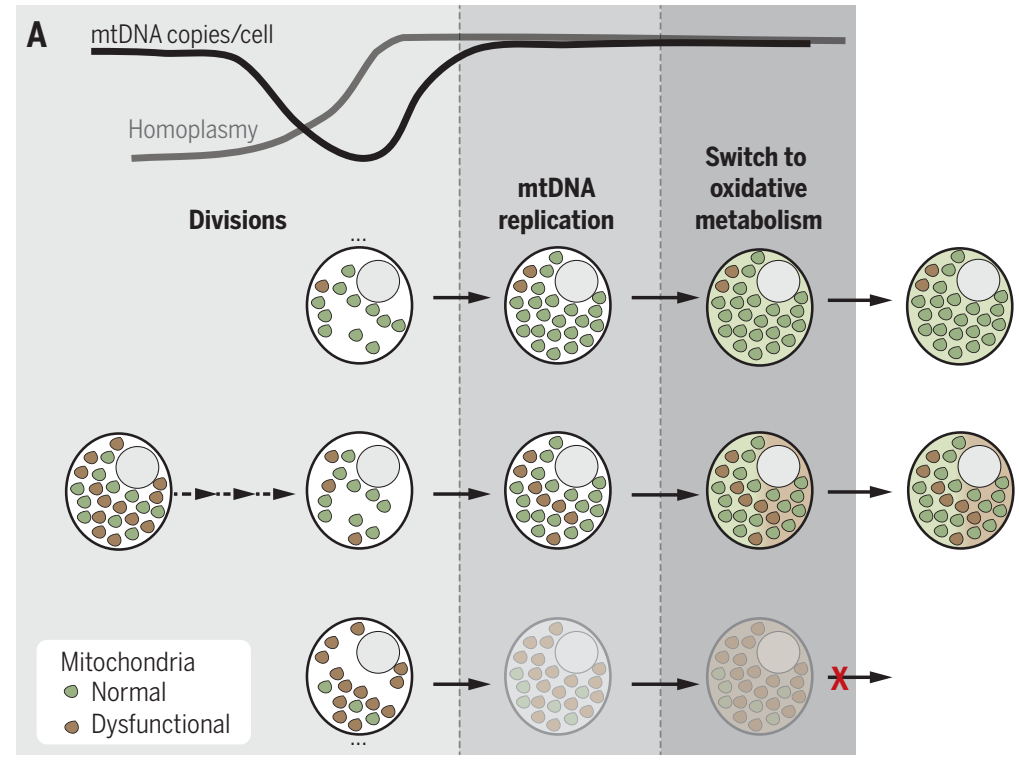

B

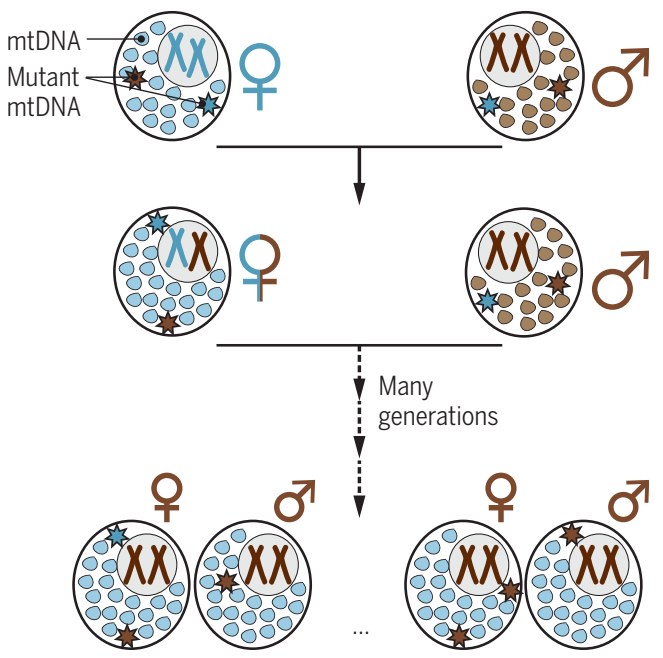

Fig. 3. Mitonuclear genomic interactions. (A) Simplified representation of the mitochondrial germline bottleneck. (B) When mtDNA and nDNA ancestries diverge, new haplotype-specific polymorphisms tend to match the nuclear ancestry rather than the mitochondrial haplotype in which they arose. [Figure adapted from Wei et al. (70)]

established, it is tempting to speculate that the molecular content of these different vesicles could trigger adaptive responses in recipient cells.

\section{Interactions between mitochondrial and nuclear genomes}

Because both the mitochondrial and nuclear genomes encode mitochondrial proteins, they have evolved functional and genetic interactions as another form of mitonuclear communication that determines mtDNA maintenance, expression, and transmission (64).

mtDNA encodes 13 subunits that play key roles in the proper function of the OXPHOS complexes (65). Despite the importance of these proteins, mtDNA has a high mutation rate that is resolved at both the organism and population levels (65). At the organism level, mechanisms exist in female germline cells to prevent transmission of deleterious mtDNA mutations to the next generation, underpinning mitochondrial germline bottleneck effects $(66,67)$ (Fig. 3A). Early primordial germ cells (PGCs) experience a profound decrease in mtDNA copy number, reducing the number of mtDNA variants in each cell. This is followed by a phase of mtDNA replication, resulting in increased level of homoplasmy in each PGC (67). The subsequent shift from glycolytic to oxidative metabolism in the PGCs acts as a soliciting test allowing expression and selection of mtDNA variants that do not impair mitochondrial function.

Because four out of five OXPHOS complexes combine subunits from mixed origins, bioenergetics are crucially affected by the compatibility of mitochondrial and nuclear OXPHOS subunits and by extension by the compatibility of the two genomes (65). Nuclear and mitochondrial genomes coevolved in populations of the same species, ultimately leading to the complementarity of mtDNA haplogroups and their nuclear background (65). This mitonuclear compatibility at the population level reflects the fact that mitonuclear genetic interactions profoundly affect mitochondrial function and whole-body physiology. In the mouse C57BL/6 strain, replacing mtDNA with that of the NZB/ OlaHsd strain triggers a limited level of stress and induces proteostasis, $\mathrm{UPR}^{\mathrm{mt}}$, and ROS signaling, ultimately remodeling mitochondrial dynamics and improving metabolism and aging (68). In humans, discordance of ancestry between nuclear and mitochondrial genomes leads to incompatibility that affects crucial markers of mitochondrial fitness such as mtDNA copy number (69). Analyzing $~ 13,000$ human whole-genome sequences demonstrated that selection of mtDNA variants is influenced by the nuclear genetic background: new polymorphisms more likely converge to match the nuclear rather than the mitochondrial genetic ancestry (Fig. 3B) (70). In other words, the nuclear genome has a selective power over mtDNA polymorphisms. mtDNA can thus be seen as an uncontrollable guest evolving in a selfish manner and eventually causing mitonuclear conflicts (71); therefore, mitonuclear genetic interactions were shaped into mechanisms guiding the fate of mtDNA, such as the germline bottleneck at the organism scale and the nucleus-imposed selection on mtDNA at the population level. A detailed understanding of mitonuclear genomic compatibility and its consequences must be sought to master the promise of mitochondrial replacement therapy (72) and to improve the genotype-specific efficacy of some treatments. Genome-wide association studies often ignore mtDNA variants (73) and mitonuclear genetic interactions, advocating for systematic investigation of mitonuclear genotypic predisposition to diseases.

\section{Conclusions and perspectives}

Mitochondria have a versatile and complex nature; they can take the appearance of a unified network or of individual units depending on the context, continuously communicating and interacting with the cellular milieu and thus challenging our vision and working models. As an example, mtDNA haplogroups are known to directly affect physiology and to be associated with diseases such as degenerative or autoimmune disorders, although the exact mechanisms remain unclear (65). The intricate links between multiple aspects of mammalian physiology and the gut microbiome are well established. The results of this review suggest that we also consider the importance of our so-called mitobiome, which is 10-fold larger than our microbiome and more complex (given that there are multiple differences in subcellular and cellular pools of mitochondria). Mitochondria should thus be thought of as small, "semiautonomous" (bacterial-like) entities that have their own genome and are occasionally shaped into populations that are able to communicate with each other, with other organelles, and even with distant tissues through a multitude of 
languages. If these mitochondrial languages are fully understood, then this will certainly unveil their central impact on homeostasis in a deeper and more integrated dimension than ever thought and would allow the development of mitochondrial medicines to treat diverse pathologies $(74,75)$.

\section{REFERENCES AND NOTES}

1. N. S. Chandel, Cell Metab. 22, 204-206 (2015),

2. S. Herzig, R. J. Shaw, Nat. Rev. Mol. Cell Biol. 19, 121-135 (2018),

3. E. Verdin, Science 350, 1208-1213 (2015),

4. L. Mouchiroud et al., Cell 154, 430-441 (2013).

5. E. Katsyuba, J. Auwerx, EMBO J. 36, 2670-2683 (2017).

6. A. P. Gomes et al., Cell 155, 1624-1638 (2013).

7. I. H. Jain et al., Science 352, 54-61 (2016).

8. L. Diebold, N. S. Chandel, Free Radic. Biol. Med. 100, 86-93 (2016).

9. N. S. Chandel et al., Proc. Natl. Acad. Sci. U.S.A. 95 11715-11720 (1998)

10. M. Schieber, N. S. Chandel, Curr. Biol. 24, R453-R462 (2014).

11. S. L. Campbell, K. E. Wellen, Mol. Cell 71, 398-408 (2018).

12. S. Nowicki, E. Gottlieb, FEBS J. 282, 2796-2805 (2015).

13. W. M. Oldham, C. B. Clish, Y. Yang, J. Loscalzo, Cell Metab. 22, 291-303 (2015).

14. S. E. Weinberg et al., Nature 565, 495-499 (2019).

15. T. Shpilka, C. M. Haynes, Nat. Rev. Mol. Cell Biol. 19, 109-120 (2018).

16. R. H. Houtkooper et al., Nature 497, 451-457 (2013).

17. T. C. Kenny, A. J. Craig, A. Villanueva, D. Germain, Cell Rep. 27. 2292-2303.e6 (2019).

18. Y. Wu et al., Cell 158, 1415-1430 (2014).

19. A. M. Nargund, M. W. Pellegrino, C. J. Fiorese, B. M. Baker, C. M. Haynes, Science 337, 587-590 (2012).

20. C. Merkwirth et al.. Cell 165, 1209-1223 (2016).

21. Y. Tian et al., Cell 165, 1197-1208 (2016).

22. C. Ma et al., Nat. Cell Biol. 21, 319-327 (2019).

23. K. Pakos-Zebrucka et al., EMBO Rep. 17, 1374-1395 (2016).
24. D. D'Amico, V. Sorrentino, J. Auwerx, Trends Biochem. Sci. 42 712-725 (2017).

25. P. M. Quirós, A. Mottis, J. Auwerx, Nat. Rev. Mol. Cell Biol. 17, 213-226 (2016).

26. M. D. Cardamone et al., Mol. Cell 69, 757-772.e7 (2018).

27. I. Gordaliza-Alaguero, C. Cantó, A. Zorzano, EMBO Rep. 20 e47928 (2019)

28. H. Wu, P. Carvalho, G. K. Voeltz, Science 361 , eaan5835 (2018)

29. Y. C. Wong, S. Kim, W. Peng, D. Krainc, Trends Cell Biol. 29 500-513 (2019)

30. S. Pickles, P. Vigié, R. J. Youle, Curr. Biol. 28, R170-R185 (2018).

31. L. J. Eichner et al., Cell Metab. 29, 285-302.e7 (2019).

32. L. Fernández-Mosquera et al., Sci. Rep. 7, 45076 (2017).

33. C. L. Nezich, C. Wang, A. I. Fogel, R. J. Youle, J. Cell Biol. 210 , 435-450 (2015)

34. N. Plotegher, M. R. Duchen, Trends Mol. Med. 23, 116-134 (2017).

35. V. Sorrentino et al.. Nature 552, 187-193 (2017).

36. D. Ryu et al., Nat. Med. 22, 879-888 (2016).

37. P. A. Andreux et al., Nat. Metab. 1, 595-603 (2019).

38. E. F. Fang et al., Nat. Neurosci. 22, 401-412 (2019).

39. K. A. Bohnert, C. Kenyon, Nature 551, 629-633 (2017).

40. M. T. Couvillion, I. C. Soto, G. Shipkovenska, L. S. Churchman, Nature 533, 499-503 (2016).

41. C. U. Mårtensson et al., Nature 569, 679-683 (2019).

42. H. Weidberg, A. Amon, Science 360, eaan4146 (2018).

43. L. Ruan et al., Nature 543, 443-446 (2017).

44. L. L. Lackner, Trends Cell Biol. 29, 580-590 (2019).

45. I. Y. Benador et al., Cell Metab. 27, 869-885.e6 (2018)

46. P. Katajisto et al., Science 348, 340-343 (2015)

47. M.-J. Wu et al., Cell Metab. 29, 993-1002.e6 (2019).

48. A. P. West, G. S. Shadel, Nat. Rev. Immunol. 17, 363-375 (2017).

49. D. A. Sliter et al., Nature 561, 258-262 (2018).

50. A. Dhir et al., Nature 560, 238-242 (2018).

51. Z. Zhong et al., Nature 560, 198-203 (2018).

52. E. L. Mills, B. Kelly, L. A. J. O'Neill, Nat. Immunol. 18, 488-498 (2017)

53. D. Matheoud et al., Nature 571, 565-569 (2019).

54. H. K. Chung et al., J. Cell Biol. 216, 149-165 (2017).

55. Q. Zhang et al., Cell 174, 870-883.e17 (2018).
56. S.-J. Kim, J. Xiao, J. Wan, P. Cohen, K. Yen, J. Physiol. 595 6613-6621 (2017).

57. A. Sugiura, G. L. McLelland, E. A. Fon, H. M. McBride, EMBO J. 33, 2142-2156 (2014)

58. D. Torralba et al., Nat. Commun. 9, 2658 (2018).

59. D. Torralba, F. Baixauli, F. Sánchez-Madrid, Front. Cell Dev. Biol. 4, 107 (2016)

60. V. J. J. Cadete et al., J. Physiol. 594, 5343-5362 (2016).

61. K. Hayakawa et al., Nature 535, 551-555 (2016).

62. C. H. Davis et al., Proc. Natl. Acad. Sci. U.S.A. 111, 9633-9638 (2014).

63. I. Melentijevic et al., Nature 542, 367-371 (2017)

64. D. B. Sloan et al., Nat. Rev. Genet. 19, 635-648 (2018).

65. D. C. Wallace, Cell 163, 33-38 (2015).

66. V. I. Floros et al., Nat. Cell Biol. 20, 144-151 (2018)

67. J. B. Stewart, P. F. Chinnery, Nat. Rev. Genet. 16, 530-542 (2015)

68. A. Latorre-Pellicer et al., Nature 535, 561-565 (2016).

69. A. A. Zaidi, K. D. Makova, Nat. Ecol. Evol. 3, 213-222 (2019)

70. W. Wei et al.. Science 364, eaau6520 (2019).

71. J. C. Havird et al., Curr. Biol. 29, R496-R511 (2019).

72. D. C. Wallace, Nat. Genet. 50, 1642-1649 (2018).

73. G. Pesole et al., EMBO Rep. 13, 473-474 (2012).

74. P. A. Andreux, R. H. Houtkooper, J. Auwerx, Nat. Rev. Drug Discov. 12, 465-483 (2013)

75. M. P. Murphy, R. C. Hartley, Nat. Rev. Drug Discov. 17, 865-886 (2018)

\section{ACKNOWLEDGMENTS}

We thank members of the Auwerx lab, in particular A. Bachmann, as well as N. Chandel and J. A. Enríquez, for their helpful comments on the manuscript. We apologize for the omission of many relevant studies due to space constraints. Funding: The work in the laboratory of the authors is supported by the EPFL, the European Research Council (ERC-AdG-787702), and the Swiss National Science Foundation (31003A-179435). Competing interests: J.A. is a founder of Mitobridge, which develops mitochondrial therapeutics.

10.1126/science.aax3768 


\section{Science}

\section{Mitocellular communication: Shaping health and disease}

Adrienne Mottis, Sébastien Herzig and Johan Auwerx

Science 366 (6467), 827-832

DOI: $10.1126 /$ science.aax3768

ARTICLE TOOLS

http://science.sciencemag.org/content/366/6467/827

RELATED

http://science.sciencemag.org/content/sci/366/6467/816.full $\mathrm{http}: / /$ science.sciencemag.org/content/sci/366/6467/818.full $\mathrm{http}: / /$ science.sciencemag.org/content/sci/366/6467/822.full http://science.sciencemag.org/content/sci/366/6467/797 full http://science.sciencemag.org/content/sci/366/6467/802.full http://science.sciencemag.org/content/sci/366/6467/843.full

REFERENCES

This article cites 75 articles, 13 of which you can access for free http://science.sciencemag.org/content/366/6467/827\#BIBL

PERMISSIONS

http://www.sciencemag.org/help/reprints-and-permissions

Use of this article is subject to the Terms of Service

Science (print ISSN 0036-8075; online ISSN 1095-9203) is published by the American Association for the Advancement of Science, 1200 New York Avenue NW, Washington, DC 20005. The title Science is a registered trademark of AAAS.

Copyright (C) 2019 The Authors, some rights reserved; exclusive licensee American Association for the Advancement of Science. No claim to original U.S. Government Works 